\title{
POLE ASSIGNMENT FOR A VIBRATING SYSTEM WITH AERODYNAMIC EFFECT*
}

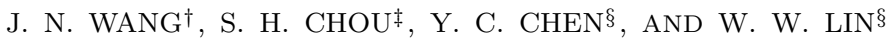

\begin{abstract}
This paper deals with a pole assignment problem by single-input state feedback control arising from a one-dimensional vibrating system with aerodynamic effect. On the practical side, we derive explicit formulae for the required controlling force terms, which can reassign part of the spectrum to the desired values while leaving the remaining spectrum unchanged. On the mathematical side, unlike the classical Sturm-Liouville problem, our eigenvalue problem is associated with a cubic pencil with unbounded operators as coefficients and has many interesting new features, one of which is that a new controllability condition appears. This condition together with the known controllability condition in the quadratic case are necessary and sufficient. This sheds light on the adjustment of the model parameters. We also analyze the spectrum of the associated noncompact operator and in particular show that the discrete spectrums of controlled and uncontrolled systems lie outside a closed interval on the negative real axis.
\end{abstract}

Key words. vibrating system, aerodynamic effect, state feedback control, pole assignment

AMS subject classifications. 93B55, 93B52

DOI. $10.1137 / \mathrm{S} 0363012902411568$

1. Introduction. Consider a vibrating system whose displacement $u=u(x, t)$ is governed by the initial boundary value problem

$$
\begin{aligned}
& \partial_{x}\left[p_{1}(x) \partial_{x} u+p_{2}(x) \mathcal{W}\left(\partial_{x} u\right)\right]-q(x) \partial_{t}^{2} u=0, \quad 0<x<L, t>0, \\
& u(0, t)=u(L, t)=0
\end{aligned}
$$

with proper initial conditions, where $p_{1}(x), p_{2}(x)$, and $q(x)$ are real-valued positive functions, and $\mathcal{W}$ is an integral operator defined by

$$
\mathcal{W} v(x, t)=\rho v+\rho \int_{0}^{t} e^{\omega(t-s)} v(x, s) d s
$$

for any complex-valued function $v:[0, L] \times[0, \infty) \rightarrow \mathbb{C}$, where $\rho \neq 0$ and $\omega \neq 0$ are real constants. The function $\mathcal{W}\left(\partial_{x} u\right)$ is called the Wagner lift-growth buildup function accounting for some dynamic effect. If $\mathcal{W}$ is zero, we recover the familiar lateral vibrating string or longitudinal vibrating rod case, depending on the boundary conditions. The more general nonzero case, i.e., the vibrating system with aerodynamic effect incorporated, has its origin in a dynamic loads analysis system (DYLOFLEX) [1].

Applying (1.2) to (1.1) with $v=\partial_{x} u$, multiplying (1.1) by $e^{-\omega t}$, and then differentiating with respect to $t$, we obtain the third order differential system

$$
\begin{aligned}
& \partial_{x}\left(\alpha(x) \partial_{x} u\right)+\partial_{x}\left(\beta(x) \partial_{x} \partial_{t} u\right)+\omega q(x) \partial_{t}^{2} u-q(x) \partial_{t}^{3} u=0, \\
& u(0, t)=u(L, t)=0,
\end{aligned}
$$

* Received by the editors July 18, 2002; accepted for publication (in revised form) August 30, 2003; published electronically February 18, 2004.

http://www.siam.org/journals/sicon/42-6/41156.html

$\dagger$ Department of Mathematics, National Taiwan University, Taipei, Taiwan (jnwang@math.ntu. edu.tw). This author was supported by NSC, Taiwan.

${ }^{\ddagger}$ Department of Mathematics and Statistics, Bowling Green State University Bowling Green, OH 43403 (chou@bgnet.bgsu.edu). This author was supported by NCTS, Taiwan, and in part by NSF under DMS-0074259.

$\S$ Department of Mathematics, National Tsing Hua University, Hsinchu, 30043, Taiwan (g893260@ oz.nthu.edu.tw, wwlin@math.nthu.edu.tw). The fourth author was supported by NSC, Taiwan. 
where

$$
\begin{aligned}
& \alpha(x)=(1-\omega) \rho p_{2}(x)-\omega p_{1}(x), \\
& \beta(x)=p_{1}(x)+\rho p_{2}(x) .
\end{aligned}
$$

To look for vibration modes, we substitute the form $u(x, t)=\phi(x) e^{\lambda t}, \lambda \in \mathbb{C}$, into (1.3) and obtain the eigenvalue problem

$$
\begin{aligned}
& \mathcal{L}(x, D, \lambda) \phi:=\left(\alpha(x) \phi^{\prime}\right)^{\prime}+\lambda\left(\beta(x) \phi^{\prime}\right)^{\prime}+\lambda^{2} \omega q(x) \phi-\lambda^{3} q(x) \phi=0 \\
& \phi(0)=\phi(L)=0
\end{aligned}
$$

where $D={ }^{\prime}=\frac{d}{d x}$. Unlike the classical Sturm-Liouville eigenvalue problem, this problem is cubic in $\lambda$. To the best of our knowledge we have not seen such a formulation before. The main purpose of this paper is to study the pole assignment associated with this problem, using the state feedback control function $b(x)$. Namely, we look at the controlled system

$$
\partial_{x}\left[p_{1}(x) \partial_{x} v+p_{2}(x) \mathcal{W}\left(\partial_{x} v\right)\right]-q(x) \partial_{t}^{2} v=b(x) w(t), \quad 0<x<L, t>0,
$$

(1.6) $v(0, t)=v(L, t)=0$,

where the feedback control force $w(t)$ has the form

$$
w(t)=\int_{0}^{L}\left[f_{1}(x) \partial_{t} v(x, t)+f_{2}(x) v(x, t)+g_{1}(x) \tilde{\mathcal{W}}\left(\partial_{x} v\right)(x, t)+g_{2}(x) \tilde{\mathcal{W}}(v)(x, t)\right] d x
$$

with

$$
\tilde{\mathcal{W}}(v)(x, t)=\rho \int_{0}^{t} e^{\omega(t-s)} v(x, s) d s .
$$

Note that here the first term $\rho v$ in the definition (1.2) has been absorbed into the first two terms on the right side of (1.7). Now substitution of $v(x, t)=\psi(x) e^{\lambda t}$ as done previously yields the eigenvalue problem associated with the controlled problem:

$$
\begin{aligned}
\mathcal{L}_{c}(x, D, \lambda) \psi:= & \left(\alpha(x) \psi^{\prime}\right)^{\prime}+\lambda\left(\beta(x) \psi^{\prime}\right)^{\prime}+\lambda^{2} \omega q(x) \psi-\lambda^{3} q(x) \psi \\
& -b \int_{0}^{L} \lambda\left(\lambda f_{1}+f_{2}\right) \psi-\omega\left(\lambda f_{1}+f_{2}\right) \psi+\rho g_{1} \psi^{\prime}+\rho g_{2} \psi d x=0, \\
\psi(0, t)= & \psi(L, t)=0 .
\end{aligned}
$$

From now on, we write $\mathcal{L}$ or $\mathcal{L}(\lambda)$ for $\mathcal{L}(x, D, \lambda)$ when no confusion can arise. Similar notation is also used for $\mathcal{L}_{c}(x, D, \lambda)$. Before investigating the pole assignment problem for (1.1), we first analyze its spectrum structure. Let the function $\alpha(x)$ in (1.4) be continuously differentiable and $\alpha(x) \neq 0$ for all $x$ in the interval $[0, L]$. For definiteness, we assume that $\alpha$ is positive throughout the whole interval. The function $\beta=\beta(x)>0$ is continuously differentiable. Then it is shown using the analytic Fredholm theorem [13] (cf. Theorem 3.3 and Remark 3.1) that the operator pencil $\mathcal{L}$ has only discrete spectrum in $\mathbb{C} \backslash E:=E^{c}$, where

$$
E:=\left[-\max _{0 \leq x \leq L} \frac{\alpha(x)}{\beta(x)},-\min _{0 \leq x \leq L} \frac{\alpha(x)}{\beta(x)}\right] .
$$


Since the interval $E$ lies in the negative real axis, we are mainly concerned with relocating discrete spectrum or poles of $(1.5)$ in $E^{c} \backslash(-\infty, 0)$. More precisely, let the discrete spectrum of $\mathcal{L}$ in $E^{c}$ be $\left\{\lambda_{1}, \ldots, \lambda_{\ell}, \lambda_{\ell+1}, \ldots\right\}$ and the first $\ell$ poles $\left\{\lambda_{1}, \ldots, \lambda_{\ell}\right\}$ be distinct, closed under complex conjugation, and furthermore $\left\{\lambda_{1}, \ldots, \lambda_{\ell}\right\} \cap\left\{\lambda_{\ell+1}, \ldots\right\}=$ $\emptyset$. Our goal is to replace $\left\{\lambda_{1}, \ldots, \lambda_{\ell}\right\}$ by $\left\{\mu_{1}, \ldots, \mu_{\ell}\right\}$, which is a conjugate set of distinct complex values in $E^{c}$, with $\left\{\mu_{1}, \ldots, \mu_{\ell}\right\} \cap\left\{\lambda_{1}, \lambda_{2}, \ldots\right\}=\emptyset$. It turns out under suitable controllability conditions that we can find explicitly the functions $f_{1}, f_{2}, g_{1}$, and $g_{2}$ so that $\left\{\lambda_{1}, \ldots, \lambda_{\ell}\right\}$ are replaced by $\left\{\mu_{1}, \ldots, \mu_{\ell}\right\}$ and other poles $\left\{\lambda_{\ell+1}, \lambda_{\ell+2}, \ldots\right\}$ remain unchanged. In Theorem 4.1, the reader can find the following formulae for the above functions with $\phi_{j}$ being the eigenfunction:

$$
\begin{aligned}
& f_{1}(x)=q \sum_{j=1}^{\ell} \xi_{j} \phi_{j}, \\
& f_{2}(x)=q \sum_{j=1}^{\ell} \xi_{j} \lambda_{j} \phi_{j}, \\
& g_{1}(x)=\left(\frac{\beta}{\rho}\right) \sum_{j=1}^{\ell} \xi_{j} \phi_{j}^{\prime}, \\
& g_{2}(x)=\left(\frac{q}{\rho}\right) \sum_{j=1}^{\ell} \xi_{j} \lambda_{j}^{2} \phi_{j},
\end{aligned}
$$

where

$$
\xi_{j}=\frac{\lambda_{j}-\mu_{j}}{\int_{0}^{L} b \phi_{j} d x} \prod_{r=1, r \neq j}^{\ell} \frac{\lambda_{j}-\mu_{r}}{\lambda_{j}-\lambda_{r}}
$$

and

$$
\int_{0}^{L} b \phi_{j} \neq 0
$$

for $j=1,2, \ldots, \ell$. Note that $(1.10)$ can be seen as a continuous version of the usual controllability condition in the matrix (discrete) case (see, for example, [3]). However, unlike the matrix case, (1.10) alone does not guarantee that $\lambda_{j}$ is a controllable mode. As a matter of fact, for the continuous case, we need to define an additional controllability condition

$$
\left(3 \lambda_{j}^{2}-2 \omega \lambda_{j}\right) \int_{0}^{L} q(x) \phi_{j}(x) \phi_{j}(x) d x+\int_{0}^{L} \beta(x) \phi_{j}^{\prime}(x) \phi_{j}^{\prime}(x) d x \neq 0
$$

for any $1 \leq j \leq \ell$. With the help of the two controllability conditions (1.10) and (1.11), we show in Theorem 4.2 that outside $E$ no extra discrete spectrum of $\mathcal{L}_{c}$ are generated except those poled. That is, the discrete spectrum of $\mathcal{L}_{c}$ in $E^{c}$ is precisely described by $\left\{\mu_{1}, \ldots, \mu_{\ell}, \lambda_{\ell+1}, \ldots\right\}$. To further refine the answer to the pole assignment problem for (1.1), we also prove that the essential spectrum of $\mathcal{L}_{c}$ is identical to that of $\mathcal{L}$, i.e., the essential spectrum of $\mathcal{L}$ does not change in the course of state feedback control. This property is, roughly, due to the fact that $\mathcal{L}_{c}$ is a compact perturbation of $\mathcal{L}$. Finally, in order for the controlled system to be realizable, the functions $f_{1}, f_{2}, g_{1}$, and $g_{2}$ need to be real, which is shown in Theorem 4.4. 
The pole assignment problem, which is concerned with assigning all eigenvalues to desired locations, is a well known and important problem in control theory. It has been extensively studied for the linear system with state or output feedback control. We refer to [16] for the detailed description of this problem and to [2] for the state-of-theart numerical methods. Our problem here is a variant of the pole assignment problem called the partial pole assignment problem, which is concerned with assigning some eigenvalues to desired positions and keeping all other eigenvalues unchanged. The partial pole assignment is more practical than the pole assignment problem, especially for distributed parameter models where we encounter infinitely many eigenvalues. The most studied distributed parameter model in this respect is the vibrating system (see, for example, [10]). The partial pole assignment problem with state feedback control for the usual vibrating distributed parameter system has been considered in [5], [6], [7], [12]. It should be noted that systems in the matrix formulation can be treated as approximations of distributed parameter systems by finite-difference or finite-element methods. In this setting, the problem becomes finite-dimensional.

This paper is partly motivated by the article [12], which considered the partial pole assignment for the vibrating rod without aerodynamic effect $(\mathcal{W}=0$ in (1.1)). The resulting equation is a standard Sturm-Liouville type. Therefore, the spectrum consists of only the discrete spectrum (i.e., eigenvalues). In [12], an explicit solution to the partial pole assignment problem with suitable state feedback was constructed and the conditions under which this solution is unique were determined. Before [12], by the similar state feedback law, Russell [15] considered the (full) pole assignment for a class of hyperbolic distributed parameter control systems. Another motivation of this paper is the partial pole assignment problem for a discrete version of (1.1) [11]. In [11], we dealt with a cubic matrix pencil rather than an operator pencil. The similar pole assignment problem for the quadratic matrix pencil was studied in [3], [4].

In the presence of aerodynamic effect, the equation in (1.1) is a Volterra integrodifferential type. This type of equation also arises in modelling phenomena involving viscoelasticity. Our pole assignment method can be used to stabilize the vibrating system with aerodynamic effect by suitable state feedback control. From the perspective of the stabilization by state feedback control, some related results for Volterra integrodifferential equations were obtained in [8], where the authors considered exponential stabilization of an abstract linear Volterra integrodifferential equation in a Hilbert space

$$
u^{\prime \prime}=-E_{1} A u(t)+E_{2} \int_{0}^{t} k(t-s) A u(s) d s+f(t)
$$

by a state feedback control given as

$$
f(t)=-C_{0} u(t)-C_{1} u^{\prime}(t),
$$

where $A$ is a positive semidefinite self-adjoint unbounded operator, $E_{1}, E_{2}$ are positive constants, and $C_{0}, C_{1}$ are bounded linear operators of finite rank. Here $k$ is a nonnegative, convex, and exponentially decreasing function with finite value at 0 . Besides considering a simpler system and having different viewpoints from ours, [8] used techniques from semigroup theory. The similar problem was also considered in [9] with a slightly different system.

2. Elementary properties of the eigensystem. In this section, we will derive some general properties of the eigenstructure of (1.5). In mathematical formalism, 
the cubic eigenvalue problem is to find a complex number $\lambda$ and a complex function $\phi$ such that

$$
\begin{aligned}
\mathcal{L}(\lambda) \phi & =0, \\
\phi(0) & =\phi(L)=0 .
\end{aligned}
$$

Assume for the time being that the eigenpairs (2.1) and (2.2) exist and that the eigenmodes are $C^{1}$ functions.

THEOREM 2.1 (dimension of eigenspace). Let $\lambda$ be an eigenvalue of (1.5). Then the dimension of the eigenspace corresponding to $\lambda$ is one, provided $(\alpha(x)+\lambda \beta(x)) \neq 0$ identically.

Proof. Let $\{\lambda, \phi\}$ and $\{\lambda, \psi\}$ be eigenpairs of the eigenvalue problem (1.5), where $\phi$ and $\psi$ are smooth. Then we have

$$
\begin{aligned}
& \mathcal{L} \phi=\left(\alpha(x) \phi^{\prime}\right)^{\prime}+\lambda\left(\beta(x) \phi^{\prime}\right)^{\prime}+\lambda^{2} \omega q(x) \phi-\lambda^{3} q(x) \phi=0, \\
& \mathcal{L} \psi=\left(\alpha(x) \psi^{\prime}\right)^{\prime}+\lambda\left(\beta(x) \psi^{\prime}\right)^{\prime}+\lambda^{2} \omega q(x) \psi-\lambda^{3} q(x) \psi=0 .
\end{aligned}
$$

Subtracting $\phi \mathcal{L} \psi$ from $\psi \mathcal{L} \phi$, we get

$$
\left(\alpha(x) \phi^{\prime}\right)^{\prime} \psi+\lambda\left(\beta(x) \phi^{\prime}\right)^{\prime} \psi-\left(\alpha(x) \psi^{\prime}\right)^{\prime} \phi-\lambda\left(\beta(x) \psi^{\prime}\right)^{\prime} \phi=0,
$$

which implies

$$
\frac{d}{d x}\left((\alpha(x)+\lambda \beta(x))\left(\phi^{\prime} \psi-\phi \psi^{\prime}\right)\right)=0 .
$$

Using the boundary conditions, we have

$$
(\alpha(x)+\lambda \beta(x))\left(\phi^{\prime} \psi-\phi \psi^{\prime}\right)=0, \quad 0<x<L .
$$

Noting that $(\alpha(x)+\lambda \beta(x)) \neq 0$ and $\phi$, and that $\psi$ are continuously differentiable, we conclude that the Wronskian

$$
\phi^{\prime} \psi-\phi \psi^{\prime}=0,
$$

and hence the functions $\phi$ and $\psi$ are dependent. This implies that the dimension of the eigenspace corresponding to $\lambda$ is one.

THEOREM 2.2 (orthogonality relation). Given two complex functions $f$ and $g$, we define

$$
\langle f, g\rangle:=\int_{0}^{L} f(x) g(x) d x .
$$

Let $\left\{\lambda_{m}, \phi_{m}\right\}$ and $\left\{\lambda_{n}, \phi_{n}\right\}$ be two distinct eigenpairs, i.e., $\lambda_{m} \neq \lambda_{n}$. Then we have the following relation:

$$
\left(\left(\lambda_{m}^{2}+\lambda_{m} \lambda_{n}+\lambda_{n}^{2}\right)-\omega\left(\lambda_{m}+\lambda_{n}\right)\right)\left\langle q \phi_{m}, \phi_{n}\right\rangle+\left\langle\beta \phi_{m}^{\prime}, \phi_{n}{ }^{\prime}\right\rangle=0 .
$$

Proof. Note that

$$
\begin{aligned}
\mathcal{L}\left(\lambda_{m}\right) \phi_{m} & =\left(\alpha(x) \phi_{m}^{\prime}\right)^{\prime}+\lambda_{m}\left(\beta(x) \phi_{m}^{\prime}\right)^{\prime}+\lambda_{m}^{2} \omega q(x) \phi_{m}-\lambda_{m}^{3} q(x) \phi_{m}=0, \\
\mathcal{L}\left(\lambda_{n}\right) \phi_{n} & =\left(\alpha(x) \phi_{n}^{\prime}\right)^{\prime}+\lambda_{n}\left(\beta(x) \phi_{n}^{\prime}\right)^{\prime}+\lambda_{n}^{2} \omega q(x) \phi_{n}-\lambda_{n}^{3} q(x) \phi_{n}=0, \\
\phi_{m}(0) & =\phi_{n}(0)=\phi_{m}(L)=\phi_{n}(L)=0 .
\end{aligned}
$$


Then from the fact that

$$
\phi_{n} \mathcal{L}\left(\lambda_{m}\right) \phi_{m}-\phi_{m} \mathcal{L}\left(\lambda_{n}\right) \phi_{n}=0
$$

we can deduce

$$
\begin{aligned}
\left(\alpha(x) \phi_{m}^{\prime}\right)^{\prime} \phi_{n}+ & \lambda_{m}\left(\beta(x) \phi_{m}^{\prime}\right)^{\prime} \phi_{n}-\left(\alpha(x) \phi_{n}^{\prime}\right)^{\prime} \phi_{m}-\lambda_{n}\left(\beta(x) \phi_{n}^{\prime}\right)^{\prime} \phi_{m} \\
& +\left(\lambda_{m}^{2}-\lambda_{n}^{2}\right) \omega q(x) \phi_{m} \phi_{n}-\left(\lambda_{m}^{3}-\lambda_{n}^{3}\right) q(x) \phi_{m} \phi_{n}=0,
\end{aligned}
$$

or equivalently

$$
\begin{aligned}
\frac{d}{d x}\left(\alpha ( x ) \left(\phi_{m}^{\prime} \phi_{n}-\right.\right. & \left.\left.\phi_{n}^{\prime} \phi_{m}\right)\right)+\frac{d}{d x}\left(\beta(x)\left(\lambda_{m} \phi_{m}^{\prime} \phi_{n}-\lambda_{n} \phi_{n}^{\prime} \phi_{m}\right)\right) \\
& -\left(\lambda_{m}-\lambda_{n}\right) \beta(x) \phi_{m}^{\prime} \phi_{n}^{\prime}+\left(\lambda_{m}^{2}-\lambda_{n}^{2}\right) \omega q(x) \phi_{m} \phi_{n} \\
& -\left(\lambda_{m}^{3}-\lambda_{n}^{3}\right) q(x) \phi_{m} \phi_{n}=0
\end{aligned}
$$

Now integrating over the interval $[0, L]$ leads to

$$
\begin{gathered}
\int_{0}^{L} \frac{d}{d x}\left(\alpha(x)\left(\phi_{m}^{\prime} \phi_{n}-\phi_{n}^{\prime} \phi_{m}\right)\right) d x+\int_{0}^{L} \frac{d}{d x}\left(\beta(x)\left(\lambda_{m} \phi_{m}^{\prime} \phi_{n}-\lambda_{n} \phi_{n}^{\prime} \phi_{m}\right)\right) d x \\
-\int_{0}^{L}\left(\lambda_{m}-\lambda_{n}\right) \beta(x) \phi_{m}^{\prime} \phi_{n}^{\prime} d x+\int_{0}^{L}\left(\lambda_{m}^{2}-\lambda_{n}^{2}\right) \omega q(x) \phi_{m} \phi_{n} d x \\
-\int_{0}^{L}\left(\lambda_{m}^{3}-\lambda_{n}^{3}\right) q(x) \phi_{m} \phi_{n} d x=0,
\end{gathered}
$$

which, upon using the boundary conditions and cancelling the nonzero common factor $\lambda_{m}-\lambda_{n}$, gives

$$
\left(\left(\lambda_{m}^{2}+\lambda_{m} \lambda_{n}+\lambda_{n}^{2}\right)-\omega\left(\lambda_{m}+\lambda_{n}\right)\right) \int_{0}^{L} q(x) \phi_{n} \phi_{m} d x+\int_{0}^{L} \beta(x) \phi_{m}^{\prime} \phi_{n}^{\prime} d x=0 .
$$

Note that the product in (2.5) is not really an inner product. Nevertheless we can think of (2.6) as an orthogonality relation for the eigenvalue system (1.5). From the proof we can view it as a generalization of Green's identity in the Sturm-Liouville eigenvalue problem to the present cubic eigenvalue problem.

3. Analysis of the spectrum of $\mathcal{L}$. Let us write down the operator pencil associated with (1.5):

$$
\mathcal{L}(x, D, \lambda)=P_{0}(x, D)+\lambda P_{1}(x, D)+\lambda^{2} P_{2}(x, D)+\lambda^{3} P_{3}(x, D),
$$

where

$$
\begin{aligned}
& P_{0}(x, D) \phi=\left(\alpha(x) \phi^{\prime}\right)^{\prime}, \\
& P_{1}(x, D) \phi=\left(\beta(x) \phi^{\prime}\right)^{\prime}, \\
& P_{2}(x, D) \phi=\omega q(x) \phi, \\
& P_{3}(x, D) \phi=-q(x) \phi .
\end{aligned}
$$

Recall that $\alpha(x)$ and $\beta(x)$ are positive $C^{1}$ functions on the closure of $\Omega:=(0, L)$. In this section we want to analyze the spectrum structure of $\mathcal{L}$ as an unbounded operator in $L^{2}(\Omega)$ with domain $\operatorname{Dom}(\mathcal{L}):=H^{2}(\Omega) \cap H_{0}^{1}(\Omega) \subset L^{2}(\Omega)$ and range $L^{2}(\Omega)$. We need a few definitions first. 
Definition 3.1. We say that $\lambda \in r(\mathcal{L})$, the resolvent set of $\mathcal{L}$, if $\mathcal{L}^{-1}(\lambda)$ exists and is bounded on $L^{2}(\Omega)$.

Definition 3.2. The set $\sigma(\mathcal{L})=\mathbb{C} \backslash r(\mathcal{L})$ is called the spectrum of $\mathcal{L}$. To further classify the spectrum of $\mathcal{L}$, we define $\lambda_{0} \in \sigma_{\text {disc }}(\mathcal{L})$, the discrete spectrum (eigenvalues) of $\mathcal{L}$, if $\lambda_{0}$ is an isolated point of $\sigma(\mathcal{L})$ and the Laurent expansion of $\mathcal{L}^{-1}$ near $\lambda_{0}$ can be written as

$$
\mathcal{L}^{-1}(\lambda)=\sum_{j=-k}^{\infty} A_{j}\left(\lambda-\lambda_{0}\right)^{j},
$$

where $0 \leq k<\infty$ and the coefficients $A_{-k}, \ldots, A_{-1}$ are all finite rank operators. The essential spectrum of $\mathcal{L}$, denoted by $\sigma_{\text {ess }}(\mathcal{L})$, is defined by $\sigma_{\text {ess }}(\mathcal{L})=\sigma(\mathcal{L}) \backslash \sigma_{\text {disc }}(\mathcal{L})$. In other words, $\mathcal{L}^{-1}$ is a meromorphic function in $\mathbb{C} \backslash \sigma_{\text {ess }}(\mathcal{L})$ and the coefficients of the negative terms in the Laurent expansion of $\mathcal{L}^{-1}$ at the point in $\sigma_{\text {disc }}(\mathcal{L})$ are finite rank operators.

Remark 3.1. If we consider the operator pencil $B=Q-\lambda$, where $Q$ is a closed operator, then the definitions of $\sigma_{\text {disc }}(B)$ and $\sigma_{\text {ess }}(B)$ in Definition 3.2 agree with the usual ones defined, for example, in [14, p. 13, p. 108]. Moreover, any isolated point of $\sigma(B)$ is in the discrete spectrum. We can also see that if $\lambda_{0} \in \sigma_{\text {disc }}(\mathcal{L})$ then the kernel of $\mathcal{L}\left(\lambda_{0}\right)$ is nontrivial. In fact, with the help of Theorem 2.1, the kernel of $\mathcal{L}\left(\lambda_{0}\right)$ is one-dimensional whenever $\lambda_{0} \in E^{c}$, where $E$ is as in (1.9).

To understand the structure of $\sigma(\mathcal{L})$, let us examine $\sigma(\mathcal{A})$, where

$$
\mathcal{A}=P_{0}+\lambda P_{1} .
$$

Intuitively, $\sigma(\mathcal{L})$ and $\sigma(\mathcal{A})$ have similar structures except discrete points, since $\mathcal{L}$, in some sense, can be treated as $\mathcal{A}$ plus compact perturbations. Note that $P_{1}^{-1}$ : $L^{2}(\Omega) \rightarrow H^{2}(\Omega)$ is bounded. Since $P_{0}$ is self-adjoint, we have that $P_{0} P_{1}^{-1}: L^{2}(\Omega) \rightarrow$ $L^{2}(\Omega)$ is a closed operator. Also, it is readily seen that $\sigma_{\text {ess }}(\mathcal{A})=\sigma_{\text {ess }}\left(P_{0} P_{1}^{-1}+\lambda\right)$ and $\sigma_{\text {disc }}(\mathcal{A})=\sigma_{\text {disc }}\left(P_{0} P_{1}^{-1}+\lambda\right)$. For consistency, here we have used the unconventional notation $\sigma_{\text {ess }}\left(P_{0} P_{1}^{-1}+\lambda\right)$ and $\sigma_{\text {disc }}\left(P_{0} P_{1}^{-1}+\lambda\right)$ to represent, respectively, the essential and discrete spectrum of $P_{0} P_{1}^{-1}$. In view of the assumptions on $\alpha(x)$ and $\beta(x)$ and the standard elliptic regularity theorem, we can see that $\sigma(\mathcal{A}) \subseteq E$, where $E$ is as in (1.5). Clearly, $E$ is an interval in the negative real axis. We are now ready to give a description of $\sigma(\mathcal{L})$.

THEOREM 3.3. Let $\alpha(x), \beta(x)$ satisfy the assumptions as stated and $q(x) \in$ $L^{\infty}(\Omega)$. Then the operator pencil $\mathcal{L}$ has only discrete spectrum in $\mathbb{C} \backslash \sigma(\mathcal{A})$.

Proof. If $\lambda \notin \sigma(\mathcal{A})$, then $\mathcal{L}^{-1}$ exists if and only if $\left(I+\left(\lambda^{2} P_{2}+\lambda^{3} P_{3}\right) \mathcal{A}^{-1}\right)^{-1}$ exists. Observe that

$$
\left(\lambda^{2} P_{2}+\lambda^{3} P_{3}\right) \mathcal{A}^{-1}=\left(\lambda^{2} P_{2}+\lambda^{3} P_{3}\right) P_{1}^{-1}\left(P_{0} P_{1}^{-1}+\lambda\right)^{-1}
$$

is compact for all $\lambda \notin \sigma(\mathcal{A})$ and is an analytic operator-valued function of $\lambda$ in $\mathbb{C} \backslash \sigma(\mathcal{A})$. In addition, we can check that $\left(I+\left(\lambda^{2} P_{2}+\lambda^{3} P_{3}\right) \mathcal{A}^{-1}\right)^{-1}$ exists at $0 \in \mathbb{C} \backslash \sigma(\mathcal{A})$. Now by the analytic Fredholm theorem [13, p. 201], we conclude that there exists a set of discrete points $S$ in $\mathbb{C} \backslash \sigma(\mathcal{A})$ such that $\left(I+\left(\lambda^{2} P_{2}+\lambda^{3} P_{3}\right) \mathcal{A}^{-1}\right)^{-1}$ exists in $\mathbb{C} \backslash(\sigma(\mathcal{A}) \cup S)$. Moreover, $\left(I+\left(\lambda^{2} P_{2}+\lambda^{3} P_{3}\right) \mathcal{A}^{-1}\right)^{-1}$ is a meromorphic of $\lambda$ in $\mathbb{C} \backslash \sigma(\mathcal{A})$ and the coefficients of the negative terms in the Laurent expansion of $\left(I+\left(\lambda^{2} P_{2}+\lambda^{3} P_{3}\right) \mathcal{A}^{-1}\right)^{-1}$ at $\lambda_{0} \in S$ are finite rank operators. In other words, points in $S$ belong to $\sigma_{\text {disc }}(\mathcal{L})$. 
Remark 3.2. It follows from Theorem 3.3 that $\mathcal{L}$ has only discrete spectrum in $E^{c}$.

Next, we want to discuss the essential spectrum of $\mathcal{L}$. It turns out that $\sigma_{\text {ess }}(\mathcal{L})$ and $\sigma_{\text {ess }}(\mathcal{A})$ are equal.

THEOREM 3.4 .

$$
\sigma_{\text {ess }}(\mathcal{L})=\sigma_{\text {ess }}(\mathcal{A})
$$

Proof. Notice that the operator

$$
\Phi(\lambda):=(\mathcal{L}-\mathcal{A}) \mathcal{A}^{-1}=\left(\lambda^{2} P_{2}+\lambda^{3} P_{3}\right) P_{1}^{-1}\left(P_{0} P_{1}^{-1}+\lambda\right)^{-1}
$$

is compact and analytic in $\mathbb{C} \backslash \sigma(\mathcal{A})$. Now if $\lambda \in \mathbb{C} \backslash \sigma(\mathcal{A})$, we can see that $\mathcal{L}^{-1}$ exists if and only if $(I+\Phi(\lambda))^{-1}$ exists. Obviously, $0 \in \mathbb{C} \backslash \sigma_{\text {ess }}(\mathcal{A})$ and $(I+\Phi(0))^{-1}=I$ exists. Recall that $\left(P_{0} P_{1}^{-1}+\lambda\right)^{-1}$ is a meromorphic function in $\mathbb{C} \backslash \sigma_{\text {ess }}(\mathcal{A})$ with finite rank residues at points in $\sigma_{\text {disc }}(\mathcal{A})$. Hence, the operator $\Phi(\lambda)$ is compact for all $\lambda \in \mathbb{C} \backslash \sigma_{\text {ess }}(\mathcal{A})$ and meromorphic in $\mathbb{C} \backslash \sigma_{\text {ess }}(\mathcal{A})$ with finite rank residues at points in $\sigma_{\text {disc }}(\mathcal{A})$. Now, by the meromorphic Fredholm theorem [14, p. 107], there exists a set of discrete points $S^{\prime}$ such that $(I+\Phi(\lambda))^{-1}$ is meromorphic in $\mathbb{C} \backslash \sigma_{\text {ess }}(\mathcal{A})$ with finite rank residues at points in $S^{\prime}$. In other words, $\mathcal{L}$ has only discrete spectrum in $\mathbb{C} \backslash \sigma_{\text {ess }}(\mathcal{A})$. Therefore, we conclude that $\sigma_{\text {ess }}(\mathcal{L}) \subseteq \sigma_{\text {ess }}(\mathcal{A})$. Conversely, by exchanging the roles of $\mathcal{L}$ and $\mathcal{A}$ and going over the same argument, we can show that $\sigma_{\text {ess }}(\mathcal{A}) \subseteq \sigma_{\text {ess }}(\mathcal{L})$.

4. Pole assignment. In this section we focus mainly on the method for replacing some particular poles of $\mathcal{L}$ in $E^{c}$ with the prescribed poles while keeping the others in $E^{c}$ unchanged. In other words, the whole concept is to determine a control force required to do such a job. We have shown that $\mathcal{L}$ has only discrete spectrum in $E^{c}$ and the kernel of $\mathcal{L}$ at this discrete spectrum is one-dimensional. Therefore, we will call $(\lambda, \phi)$ an eigenpair of $\mathcal{L}$ whenever $\mathcal{L}(\lambda) \phi=0$ for $\lambda \in E^{c}$. Suppose that $b(x)$ is a real control function, and $w(t)$ is a control force being applied to (1.1). Let $v(x, t)$ be the response of the controlled system. For our study, we take the control force $w(t)$ of the form

$$
w(t)=\int_{0}^{L}\left[f_{1}(x) \partial_{t} v(x, t)+f_{2}(x) v(x, t)+g_{1}(x) \tilde{W}\left(\partial_{x} v\right)(x, t)+g_{2}(x) \tilde{W}(v)(x, t)\right] d x,
$$

where

$$
\tilde{W}(v)(x, t)=\rho \int_{0}^{t} e^{\omega(t-s)} v(x, s) d s
$$

(We use $\tilde{W}$, since the first term $\rho$ in the function $W$ has been absorbed into other terms.) Thus, the controlled system is expressed as

$$
\begin{aligned}
& \partial_{x}\left[p_{1}(x) \partial_{x} v+p_{2}(x) W\left(\partial_{x} v\right)\right]-q(x) \partial_{t}^{2} v \\
& \quad=b(x) \int_{0}^{L}\left[f_{1}(x) v+f_{2}(x) \partial_{t} v+g_{1}(x) \tilde{W}\left(\partial_{x} v\right)+g_{2}(x) \tilde{W}(v)\right] d x \\
& v(0, t)=v(L, t)=0 .
\end{aligned}
$$


Simplifying (4.2) as done previously for the free system and letting $v(x, t)=$ $\psi(x) e^{\lambda t}$ yields

$$
\begin{gathered}
\mathcal{L}_{c}(\lambda) \psi:=\left(\alpha(x) \psi^{\prime}\right)^{\prime}+\lambda\left(\beta(x) \psi^{\prime}\right)^{\prime}+\lambda^{2} \omega q(x) \psi-\lambda^{3} q(x) \psi \\
\quad-b \int_{0}^{L}\left[\lambda\left(\lambda f_{1}+f_{2}\right) \psi-\omega\left(\lambda f_{1}+f_{2}\right) \psi+\rho g_{1} \psi^{\prime}+\rho g_{2} \psi\right] d x=0 \\
(4.3) \quad \psi(0, t)=\psi(L, t)=0 .
\end{gathered}
$$

Here we restate that $\alpha(x)$ and $\beta(x)$ belong to $C^{1}(\bar{\Omega})$, and $q(x)$ is in $L^{\infty}(\Omega)$.

Before we begin, we would like to do some simple adjustments and arrangements. Let $\left\{\lambda_{1}, \lambda_{2}, \ldots\right\}=\sigma_{\text {disc }}(\mathcal{L}) \backslash E$ with associated eigenvectors $\left\{\phi_{1}, \phi_{2}, \ldots\right\}$ and let the first $\ell$ discrete spectrum $\left\{\lambda_{1}, \ldots, \lambda_{\ell}\right\}$ satisfy $\left\{\lambda_{1}, \ldots, \lambda_{\ell}\right\} \cap\left\{\lambda_{\ell+1}, \lambda_{\ell+2}, \ldots\right\}=\emptyset$. Now we will show how to obtain the control force $w(t)$, which assigns poles $\left\{\lambda_{1}, \ldots, \lambda_{\ell}\right\}$ of $\mathcal{L}$ to prescribed values (still lying in $E^{c}$ ) while leaving the other poles in $E^{c}$ unchanged. More precisely, let $\left\{\mu_{1}, \ldots, \mu_{\ell}\right\}$ be in $E^{c}$ with $\left\{\mu_{1}, \ldots, \mu_{\ell}\right\} \cap\left\{\lambda_{1}, \lambda_{2}, \ldots\right\}=\emptyset$. Then we wish to find $f_{1}(x), f_{2}(x), g_{1}(x)$ and $g_{2}(x)$ in (4.3) such that $\left\{\mu_{1}, \ldots, \mu_{\ell}, \lambda_{\ell+1}, \ldots\right\}=$ $\sigma_{\text {disc }}\left(\mathcal{L}_{c}\right) \backslash E$.

Theorem 4.1. Let $b(x) \in L^{2}(\Omega)$ satisfy

$$
\int_{0}^{L} b \phi_{j} d x=\left\langle b, \phi_{j}\right\rangle \neq 0 \quad \text { for } j=1,2, \ldots, \ell .
$$

Assume that $\left\{\lambda_{1}, \ldots, \lambda_{\ell}\right\}$ has distinct elements and so does $\left\{\mu_{1}, \ldots, \mu_{\ell}\right\}$. Let the following functions be defined:

$$
\begin{aligned}
f_{1}(x) & :=q \sum_{j=1}^{\ell} \xi_{j} \phi_{j}, \\
f_{2}(x) & :=q \sum_{j=1}^{\ell} \xi_{j} \lambda_{j} \phi_{j}, \\
g_{1}(x) & :=\left(\frac{\beta}{\rho}\right) \sum_{j=1}^{\ell} \xi_{j} \phi_{j}^{\prime}, \\
g_{2}(x) & :=\left(\frac{q}{\rho}\right) \sum_{j=1}^{\ell} \xi_{j} \lambda_{j}^{2} \phi_{j},
\end{aligned}
$$

where

$$
\xi_{j}=\frac{\lambda_{j}-\mu_{j}}{\left\langle b, \phi_{j}\right\rangle} \prod_{r=1, r \neq j}^{\ell} \frac{\lambda_{j}-\mu_{r}}{\lambda_{j}-\lambda_{r}}, \quad j=1,2, \ldots, \ell .
$$

Then we have that

(i) $\sigma_{\text {ess }}\left(\mathcal{L}_{c}\right)=\sigma_{\text {ess }}(\mathcal{L})$ and $\mathcal{L}_{c}$ has only discrete spectrum in $E^{c}$,

(ii) $\left\{\mu_{1}, \ldots, \mu_{\ell}, \lambda_{\ell+1}, \ldots\right\} \subset \sigma_{\text {disc }}\left(\mathcal{L}_{c}\right) \backslash E$.

Proof. (i) Since $\alpha(x), \beta(x) \in C^{1}(\bar{\Omega})$ and $q(x) \in L^{\infty}(\Omega)$, we get from the elliptic regularity theorem that $\phi_{j} \in H^{2}(\Omega) \cap H_{0}^{1}(\Omega)$. Using the integration by parts in the 
integral term $\int_{0}^{L} \rho g_{1} \psi^{\prime} d x$ of (4.3), we obtain that

$$
\begin{aligned}
& b \int_{0}^{L}\left[\lambda\left(\lambda f_{1}+f_{2}\right) \psi-\omega\left(\lambda f_{1}+f_{2}\right) \psi+\rho g_{1} \psi^{\prime}+\rho g_{2} \psi\right] d x \\
& =b \int_{0}^{L}\left\{\lambda\left(\lambda f_{1}+f_{2}\right)-\omega\left(\lambda f_{1}+f_{2}\right) \psi-\rho g_{1}^{\prime}+\rho g_{2}\right\} \psi d x \\
& :=G(\lambda) \psi(x) .
\end{aligned}
$$

It is clear that $G(\lambda)$ is an integral operator depending on $\lambda$ analytically. In view of (4.5) and the fact that $b \in L^{2}(\Omega)$, we have that $G(\lambda)$ is a Hilbert-Schmidt operator on $L^{2}(\Omega)$ and hence compact. Going over the proofs of Theorems 3.3 and 3.4 once again, we immediately conclude that $\mathcal{L}_{c}$ has only discrete spectrum in $E^{c}$ and $\sigma_{\text {ess }}\left(\mathcal{L}_{c}\right)=\sigma_{\text {ess }}(\mathcal{A})=\sigma_{\text {ess }}(\mathcal{L})$.

(ii) We first show that with (4.5), for each $k \geq \ell+1$, the eigenpair $\left\{\lambda_{k}, \phi_{k}\right\}$ of $\mathcal{L}$ satisfies $\mathcal{L}_{c}\left(\lambda_{k}\right) \phi_{k}=0$. The idea is simply to show that the control term in (4.3) is zero when $\lambda$ is replaced by any $\lambda_{k}, k \geq \ell+1$. Clearly,

$$
\begin{aligned}
& \left(\alpha \phi_{k}^{\prime}\right)^{\prime}+\lambda_{k}\left(\beta \phi_{k}^{\prime}\right)^{\prime}+\lambda_{k}^{2} \omega q \phi_{k}-\lambda_{k}^{3} q \phi_{k} \\
& \quad-b \int_{0}^{L} \lambda_{k}\left(\lambda_{k} f_{1}+f_{2}\right) \phi_{k}-\omega\left(\lambda_{k} f_{1}+f_{2}\right) \phi_{k}+\rho g_{1} \phi_{k}^{\prime}+\rho g_{2} \phi_{k} d x \\
& =-b \int_{0}^{L} \lambda_{k}\left(\lambda_{k} f_{1}+f_{2}\right) \phi_{k}-\omega\left(\lambda_{k} f_{1}+f_{2}\right) \phi_{k}+\rho g_{1} \phi_{k}^{\prime}+\rho g_{2} \phi_{k} d x \\
& =-b \sum_{i=1}^{\ell} \xi_{i}\left\{\left(\left(\lambda_{k}^{2}+\lambda_{k} \lambda_{i}+\lambda_{i}^{2}\right)-\omega\left(\lambda_{k}+\lambda_{i}\right)\right)\left\langle q \phi_{k}, \phi_{i}\right\rangle+\left\langle\beta \phi_{k}^{\prime}, \phi_{i}{ }^{\prime}\right\rangle\right\} .
\end{aligned}
$$

By the orthogonality relation (2.6) we then conclude that $\mathcal{L}_{c}\left(\lambda_{k}\right) \phi_{k}=0$, i.e., $\lambda_{k} \in \sigma_{\text {disc }}\left(\mathcal{L}_{c}\right)$.

Now suppose that $\left\{\mu_{k}, \chi_{k}\right\}$ is the solution of the system

$$
\begin{aligned}
& \left(\alpha \chi_{k}^{\prime}\right)^{\prime}+\mu_{k}\left(\beta \chi_{k}^{\prime}\right)^{\prime}+\mu_{k}^{2} \omega q \chi_{k}-\mu_{k}^{3} q \chi_{k}=b, \\
& \chi_{k}(0)=\chi_{k}(L)
\end{aligned}
$$

for $1 \leq k \leq \ell$. The existence of the solution is guaranteed by the fact that $\mu_{k} \in r(\mathcal{L})$ for all $k$ and $b \in L^{2}(\Omega)$. We will now show that $\mathcal{L}_{c}\left(\mu_{k}\right) \chi_{k}=0$ for all $1 \leq k \leq \ell$. First we consider the following:

$$
\left\{\begin{array}{l}
\left(\alpha \chi_{k}^{\prime}\right)^{\prime}+\mu_{k}\left(\beta \chi_{k}^{\prime}\right)^{\prime}+\mu_{k}^{2} \omega q \chi_{k}-\mu_{k}^{3} q \chi_{k}=b \\
\chi_{k}(0)=\chi_{k}(L)=0, \quad 1 \leq k \leq \ell
\end{array}\right.
$$

and

$$
\left\{\begin{array}{l}
\left(\alpha \phi_{j}^{\prime}\right)^{\prime}+\lambda_{j}\left(\beta \phi_{j}^{\prime}\right)^{\prime}+\lambda_{j}^{2} \omega q \phi_{j}-\lambda_{j}^{3} q \phi_{j}=0 \\
\phi_{j}(0)=\phi_{j}(L)=0, \quad 1 \leq j \leq \ell
\end{array}\right.
$$

Multiplying (4.8) by $\phi_{j}$ and subtracting from it the quantity (4.9) times $\chi_{k}$, we get, on the one hand,

$$
\begin{gathered}
\left(\lambda_{j}-\mu_{k}\right)\left\langle\beta \chi_{k}^{\prime}, \phi_{j}{ }^{\prime}\right\rangle+\left(\mu_{k}^{2}-\lambda_{j}^{2}\right) \omega\left\langle q \chi_{k}, \phi_{j}\right\rangle \\
-\left(\mu_{k}^{3}-\lambda_{j}^{3}\right)\left\langle q \chi_{k}, \phi_{j}\right\rangle=\left\langle b, \phi_{j}\right\rangle .
\end{gathered}
$$


On the other hand, let $\mu \in \mathbb{C}$ and $\psi:[0, L] \rightarrow \mathbb{C}$, and define

$$
\begin{gathered}
F(\mu, \psi):=\int_{0}^{L} \mu\left(\mu f_{1}+f_{2}\right) \psi-\omega\left(\mu f_{1}+f_{2}\right) \psi+\rho g_{1} \psi^{\prime}+\rho g_{2} \psi d x \\
=\sum_{j=1}^{\ell} \xi_{j}\left[\left\langle\beta \psi^{\prime}, \phi_{j}{ }^{\prime}\right\rangle-\left(\mu+\lambda_{j}\right) \omega\left\langle q \psi, \phi_{j}\right\rangle\right. \\
\left.\quad+\left(\lambda_{j}^{2}+\mu \lambda_{j}+\mu^{2}\right)\left\langle q \psi, \phi_{j}\right\rangle\right] .
\end{gathered}
$$

Here in the notation we suppress the dependence of $F$ on $\phi_{j}$ 's.

By writing

$$
\begin{aligned}
\xi_{j} & =\left(\frac{\lambda_{j}-\mu_{j}}{\left\langle b, \phi_{j}\right\rangle} \prod_{r=1, r \neq j}^{\ell} \frac{\lambda_{j}-\mu_{r}}{\lambda_{j}-\lambda_{r}}\right) \\
& =\left(\lambda_{j}-\mu_{k}\right)\left(\frac{\prod_{r=1, r \neq k}^{\ell}\left(\lambda_{j}-\mu_{r}\right)}{\prod_{r=1, r \neq j}^{\ell}\left(\lambda_{j}-\lambda_{r}\right)}\right)\left(\frac{1}{\left\langle b, \phi_{j}\right\rangle}\right),
\end{aligned}
$$

we obtain

$$
\begin{aligned}
F\left(\mu_{k}, \psi\right)= & \sum_{j=1}^{\ell}\left(\frac{\prod_{r=1, r \neq k}^{\ell}\left(\lambda_{j}-\mu_{r}\right)}{\prod_{r=1, r \neq j}^{\ell}\left(\lambda_{j}-\lambda_{r}\right)}\right)\left(\frac{1}{\left\langle b, \phi_{j}\right\rangle}\right) \\
& \times\left[\left(\lambda_{j}-\mu_{k}\right)\left\langle\beta \psi^{\prime}, \phi_{j}{ }^{\prime}\right\rangle+\left(\mu_{k}^{2}-\lambda_{j}^{2}\right) \omega\left\langle q \psi, \phi_{j}\right\rangle\right. \\
& \left.\quad-\left(\lambda_{j}^{3}-\mu_{k}^{3}\right)\left\langle q \psi, \phi_{j}\right\rangle\right] .
\end{aligned}
$$

Let us check the validity of the equation

$$
\left(\alpha \chi_{k}^{\prime}\right)^{\prime}+\mu_{k}\left(\beta \chi_{k}^{\prime}\right)^{\prime}+\mu_{k}^{2} \omega q \chi_{k}-\mu_{k}^{3} q \chi_{k}-b F\left(\mu_{k}, \chi_{k}\right)=0 .
$$

Using (4.8), (4.10), and (4.12), we see that the left-hand side of (4.14) is

$$
b-b\left\{\sum_{j=1}^{\ell} \frac{\prod_{r=1, r \neq k}^{\ell}\left(\lambda_{j}-\mu_{r}\right)}{\prod_{r=1, r \neq j}^{\ell}\left(\lambda_{j}-\lambda_{r}\right)}\right\}=0, \quad 1 \leq k \leq \ell,
$$

where we used an identity from [3]: for any $1 \leq k \leq \ell$,

$$
\sum_{j=1}^{\ell} \frac{\prod_{r=1, r \neq k}^{\ell}\left(\lambda_{j}-\mu_{r}\right)}{\prod_{r=1, r \neq j}^{\ell}\left(\lambda_{j}-\lambda_{r}\right)}=1 .
$$

This completes the proof of (ii).

Next we want to show that the discrete spectrum of $\mathcal{L}_{c}$ in $E^{c}$ is precisely given by $\left\{\mu_{1}, \ldots, \mu_{\ell}, \lambda_{\ell+1}, \ldots\right\}$. That is, no new extra discrete spectrum occurs in $E^{c}$ except those prescribed.

TheOrem 4.2. Let $f_{1}, f_{2}, g_{1}$, and $g_{2}$ be defined as in (4.5) and (4.6), and the assumptions of Theorem 4.1 hold. Moreover, assume that $\left(\lambda_{k}, \phi_{k}\right)$ satisfies

$$
\left(3 \lambda_{k}^{2}-2 \omega \lambda_{k}\right)\left\langle q \phi_{k}, \phi_{k}\right\rangle+\left\langle\beta \phi_{k}^{\prime}, \phi_{k}^{\prime}\right\rangle \neq 0
$$


for $1 \leq k \leq \ell$. Then the discrete spectrum of $\mathcal{L}_{c}$ in $E^{c}$ is precisely given by $\left\{\mu_{1}, \ldots, \mu_{\ell}\right.$, $\left.\lambda_{\ell+1}, \ldots\right\}$, i.e.,

$$
\left\{\mu_{1}, \ldots, \mu_{\ell}, \lambda_{\ell+1}, \ldots\right\}=\sigma_{d i s c}\left(\mathcal{L}_{c}\right) \backslash E .
$$

Proof. The previous theorem shows that

$$
\left\{\mu_{1}, \ldots, \mu_{\ell}, \lambda_{\ell+1}, \ldots\right\} \subset \sigma_{d i s c}\left(\mathcal{L}_{c}\right) \backslash E .
$$

Here, we only need to prove the opposite inclusion.

We first claim that $\left\{\lambda_{1}, \ldots, \lambda_{\ell}\right\} \notin \sigma_{\text {disc }}\left(\mathcal{L}_{c}\right) \backslash E$. Equivalently, those poled will not show up again. To begin, we show that $\mathcal{L}_{c}\left(\lambda_{k}\right) \phi_{k} \neq 0$ for $1 \leq k \leq \ell$. In fact, since $\left\{\lambda_{k}, \phi_{k}\right\}$ is an eigenpair of $\mathcal{L}$, we have that

$$
\begin{aligned}
\mathcal{L}_{c}\left(\lambda_{k}\right) \phi_{k} & =\mathcal{L}\left(\lambda_{k}\right) \phi_{k}-b(x) F\left(\lambda_{k}, \phi_{k}\right) \\
& =-b(x) F\left(\lambda_{k}, \phi_{k}\right) .
\end{aligned}
$$

By virtue of (4.11) and the orthogonality relation, we can see that

$$
F\left(\lambda_{k}, \phi_{k}\right)=\xi_{k}\left\{\left(3 \lambda_{k}^{2}-2 \omega \lambda_{k}\right)\left\langle q \phi_{k}, \phi_{k}\right\rangle+\left\langle\beta \phi_{k}^{\prime}, \phi_{k}^{\prime}\right\rangle\right\} .
$$

It is clear that $\xi_{k} \neq 0$ and, therefore, $F\left(\lambda_{k}, \phi_{k}\right) \neq 0$ by the condition (4.15).

Next, we claim $\mathcal{L}_{c}\left(\lambda_{k}\right) \psi \neq 0$ for $1 \leq k \leq \ell$ if $\psi$ and $\phi_{k}$ are linearly independent. In fact, since the dimension of the eigenspace of $\mathcal{L}$ is one, we have $\mathcal{L}\left(\lambda_{k}\right) \psi \neq 0$. Suppose, on the contrary, $0=\mathcal{L}_{c}\left(\lambda_{k}\right) \psi=\mathcal{L}\left(\lambda_{k}\right) \psi-b(x) F\left(\lambda_{k}, \psi\right)$. On the one hand,

$$
\begin{aligned}
0 & =\left\langle\psi, \mathcal{L}\left(\lambda_{k}\right) \phi_{k}\right\rangle=\left\langle\mathcal{L}\left(\lambda_{k}\right) \psi, \phi_{k}\right\rangle \\
& =\left\langle b F\left(\lambda_{k}, \psi\right), \phi\right\rangle=F\left(\lambda_{k}, \psi\right)\left\langle b, \phi_{k}\right\rangle .
\end{aligned}
$$

But in view of (4.4), this leads to $F\left(\lambda_{k}, \psi\right)=0$, and hence $0=\mathcal{L}\left(\lambda_{k}\right) \psi$, a contradiction.

Finally we show that if $\mu \notin\left\{\lambda_{1}, \lambda_{2}, \ldots\right\} \cup\left\{\mu_{1}, \ldots, \mu_{k}\right\}$ and $\mu \notin E$, then $\mu \notin$ $\sigma_{\text {disc }}\left(\mathcal{L}_{c}\right) \backslash E$. We will use a contradiction. Suppose that $\{\mu, \phi\}$ is an eigenpair of $\mathcal{L}_{c}$. Thus

$$
\begin{aligned}
0 & =\mathcal{L}_{c}(\mu) \phi \\
& =\mathcal{L}(\mu) \phi-b(x) C,
\end{aligned}
$$

where the constant $C=F(\mu, \phi)$. Note that $C \neq 0$; otherwise $\mu \in \sigma_{\text {disc }}(\mathcal{L}) \backslash E$. By normalization, we can find $\{\mu, \psi\}$, where $\psi=\frac{1}{C} \phi$, so that

$$
\mathcal{L}(\mu) \psi=b .
$$

Now we can compute $F(\mu, \psi)$ as we did for $F\left(\mu_{k}, \psi\right)$ going from (4.8) to (4.13), and hence

$$
\begin{aligned}
0 & =\mathcal{L}_{c}(\mu) \psi \\
& =\mathcal{L}(\mu) \psi-b(x) F(\mu, \psi) \\
& =b-b F(\mu, \psi) \\
& =b\left[1-\sum_{j=1}^{\ell} \frac{1}{\lambda_{j}-\mu} \frac{\Pi_{r=1}^{\ell}\left(\lambda_{j}-\mu_{r}\right)}{\Pi_{r=1, r \neq j}^{\ell}\left(\lambda_{j}-\lambda_{r}\right)}\right] \\
& \neq 0,
\end{aligned}
$$


which is a contradiction since the bracketed term can be transformed into a polynomial of degree $\ell$ in $\mu$ and $\mu_{1}, \ldots, \mu_{\ell}$ have been used up as the $\ell$ roots. This completes the proof.

In view of the above, conditions (4.4) and (4.15) can be legitimately called the controllability conditions. We now show that if either one of (4.4) and (4.15) is violated, then the mode $\lambda_{j}$ cannot be relocated by our designed control force, where $1 \leq j \leq \ell$.

THEOREM 4.3. For any $1 \leq j \leq \ell$, let $\left(\lambda_{j}, \phi_{j}\right)$ satisfy either $\left\langle b, \phi_{j}\right\rangle=0$ or $\left(3 \lambda_{j}^{2}-2 \omega \lambda_{j}\right)\left\langle q \phi_{j}, \phi_{j}\right\rangle+\left\langle\beta \phi_{j}^{\prime}, \phi_{j}^{\prime}\right\rangle=0$. Then $\lambda_{j} \in \sigma_{\text {disc }}\left(\mathcal{L}_{c}\right)$, with $f_{1}, f_{2}, g_{1}$, and $g_{2}$ of $\mathcal{L}_{c}$ being given in (4.5) and (4.6).

Proof. From the proof of Theorem 4.2, we immediately see that $\mathcal{L}_{c}\left(\lambda_{j}\right) \phi_{j}=0$ if $\left(3 \lambda_{j}^{2}-2 \omega \lambda_{j}\right)\left\langle q \phi_{j}, \phi_{j}\right\rangle+\left\langle\beta \phi_{j}^{\prime}, \phi_{j}^{\prime}\right\rangle=0$. Now we assume that $\left\langle b, \phi_{j}\right\rangle=0$. In view of the form of $\mathcal{L}_{c}$, we have $\left\langle\mathcal{L}_{c}\left(\lambda_{j}\right) \psi, \phi_{j}\right\rangle=0$ for any $\psi \in H^{2}(\Omega) \cap H_{0}^{1}(\Omega)$, which implies that $\mathcal{L}_{c}\left(\lambda_{j}\right)$ is not invertible. For, if $\mathcal{L}_{c}\left(\lambda_{j}\right)^{-1}$ exists, then we can find a $\psi_{j} \in H^{2}(\Omega) \cap H_{0}^{1}(\Omega)$ such that $\mathcal{L}_{c}\left(\lambda_{j}\right) \psi_{j}=\bar{\phi}_{j}$ and therefore $\left\langle\mathcal{L}_{c}\left(\lambda_{j}\right) \psi, \phi_{j}\right\rangle \neq 0$. Since $\mathcal{L}_{c}$ has only discrete spectrum in $E^{c}, \lambda_{j}$ must be in $\sigma_{\text {disc }}\left(\mathcal{L}_{c}\right)$.

Finally, in order for the control to be realizable, we need to show that $f_{1}, f_{2}, g_{1}$, and $g_{2}$ are real functions.

TheOrem 4.4. Assume that both sets $\left\{\lambda_{1}, \ldots, \lambda_{\ell}\right\}$ and $\left\{\mu_{1}, \ldots, \mu_{\ell}\right\}$ are closed under complex conjugation. Then the functions $f_{1}, f_{2}, g_{1}$, and $g_{2}$ are real functions.

Proof. Rewriting $\xi_{j}$ yields

$$
\begin{aligned}
\xi_{j} & =\frac{\lambda_{j}-\mu_{j}}{\left\langle b, \phi_{j}\right\rangle} \prod_{r=1, r \neq j}^{\ell} \frac{\lambda_{j}-\mu_{r}}{\lambda_{j}-\lambda_{r}} \\
& =\frac{\prod_{r=1}^{\ell}\left(\lambda_{j}-\mu_{r}\right)}{\left\langle b, \phi_{j}\right\rangle \prod_{r=1, r \neq j}^{\ell}\left(\lambda_{j}-\lambda_{r}\right)} .
\end{aligned}
$$

Therefore, if $\lambda_{j}$ is real and its associated eigenfunction $\phi_{j}$ is also real, then $\bar{\xi}_{j}=\xi_{j}$, i.e., $\xi_{j}$ is real. Now assume that $\lambda_{j}$ and $\lambda_{j+1}$ are a conjugate pair and their eigenfunctions satisfy $\phi_{j}=\bar{\phi}_{j}$. Then we can see that

$$
\begin{aligned}
\xi_{j+1} & =\frac{\prod_{r=1}^{\ell}\left(\lambda_{j+1}-\mu_{r}\right)}{\left\langle b, \phi_{j+1}\right\rangle \prod_{r=1, r \neq j+1}^{\ell}\left(\lambda_{j+1}-\lambda_{r}\right)} \\
& =\frac{\prod_{r=1}^{\ell}\left(\bar{\lambda}_{j}-\mu_{r}\right)}{\left\langle b, \bar{\phi}_{j}\right\rangle \prod_{r=1, r \neq j+1}^{\ell}\left(\bar{\lambda}_{j}-\lambda_{r}\right)} \\
& =\frac{\prod_{r=1}^{\ell}\left(\bar{\lambda}_{j}-\mu_{r}\right)}{\left\langle b, \bar{\phi}_{j}\right\rangle \prod_{r=1, r \neq j}^{\ell}\left(\bar{\lambda}_{j}-\bar{\lambda}_{r}\right)} \\
& =\bar{\xi}_{j} .
\end{aligned}
$$

Thus, for $f_{1}$, we have that

$$
\bar{f}=q \sum_{j=1}^{\ell} \bar{\xi}_{j} \bar{\phi}_{j}=q \sum_{j=1}^{\ell} \xi_{j} \phi_{j}=f_{1},
$$

i.e., $f_{1}$ is real. Similarly, we can prove that $f_{2}, g_{1}$, and $g_{2}$ are real. 


\section{REFERENCES}

[1] R. Miller, R. Kroll, and R. Clemmons, Dynamic Loads Analysis System (DYLOFLEX) Summary, Tech. Rep. NASA Contractor Report 2846-1, NASA, 1979.

[2] B. N. Datta, Numerical Methods for Linear Control Systems Design and Analysis, Academic Press, New York, to appear.

[3] B. N. Datta, S. Elhay, and Y. M. Ram, Orthogonality and partial pole assignment of the symmetric definite quadratic pencil, Linear Algebra Appl., 257 (1997), pp. 29-48.

[4] B. N. Datta, S. Elhay, Y. M. Ram, and D. R. Sarkissian, Partial eigenstructure assignment for the quadratic pencil, J. Sound Vibration, 230 (2000), pp. 101-110.

[5] B. N. Datta, Y. M. Ram, and R. D. Sarkissian, Spectrum modification for gyroscopic systems, ZAMM Z. Angew. Math. Mech., 82 (2002), pp. 191-200.

[6] B. N. DATTA AND R. D. SARKissian, Feedback control in distributed parameter gyroscopic systems: A solution of the partial eigenvalue assignment problem, Mechanical Systems and Signal Processing, special issue on vibration control, 16 (2001), pp. 3-17.

[7] B. N. DatTa And R. D. Sarkissian, A computational method for feedback control in distributed parameter systems, in Proceedings of the 8th IEEE International Conference on Methods and Models in Robotics and Automation, 2002, pp. 139-144.

[8] W. Desch And R. K. Miller, Exponential stabilization of Volterra integro-differential equations in Hilbert space, J. Differential Equations, 70 (1987), pp. 366-389.

[9] W. Desch AND R. K. Miller, Exponential stabilization of Volterra integral equations with singular kernels, J. Integral Equations Appl., 1 (1988), pp. 397-433.

[10] D. J. Inman, Vibration: With Control, Measurement, and Stability, Prentice-Hall, Englewood Cliffs, NJ, 1989.

[11] W. W. LIN AND J. N. WANG, Robust partial pole assignment for the vibrating system with aerodynamic effect, Numer. Linear Algebra Appl., to appear.

[12] Y. M. RAM, Pole assignment for the vibrating rod, Quart. J. Mech. Appl. Math., 51 (1998), pp. 461-476.

[13] M. Reed And B. Simon, Methods of Modern Mathematical Physics I. Functional Analysis, Academic Press, New York, 1973.

[14] M. Reed And B. Simon, Methods of Modern Mathematical Physics IV. Analysis of Operators, Academic Press, New York, 1978.

[15] D. L. Russell, Canonical forms and spectral determination for a class of hyperbolic distributed parameter control systems, J. Math. Anal. Appl., 62 (1978), pp. 186-225.

[16] W. Wonham, Murray Linear Multivariable Control: A Geometric Approach, 3rd ed., Appl. Math. 10, Springer-Verlag, New York, 1985. 